Received: 07.03.2020

Revised: 06.04.2020

Accepted: 10.04 .2020

DOI: $10.17804 / 2410-9908.2020 .2 .061-075$

\title{
EXACT SOLUTION FOR DESCRIBING A UNIDIRECTIONAL MARANGONI FLOW OF A VISCOUS INCOMPRESSIBLE FLUID WITH THE NAVIER BOUNDARY CONDITION. PRESSURE FIELD INVESTIGATION
}

\author{
N. V. Burmasheva ${ }^{\text {a)* }}$ and E. Yu. Prosviryakov ${ }^{\text {b) }}$ \\ Institute of Engineering Science, Ural Branch of the Russian Academy of Sciences, \\ 34 Komsomolskaya St., Ekaterinburg, 620049, Russian Federation \\ a) (iD https://orcid.org/0000-0003-4711-1894 nat_burm@mail.ru; \\ b) (iD https://orcid.org/0000-0002-2349-7801 evgen_pros@mail.ru \\ *Corresponding author. E-mail: nat_burm@mail.ru \\ Address for correspondence: 34 Komsomolskaya St., Ekaterinburg, 620049, Russian Federation \\ Tel.:+7 (343) 37535 76; fax: 3745330
}

The article studies the properties of the pressure field in a unidirectional thermocapillary flow of a viscous incompressible fluid in an infinite horizontal layer of a given thickness. A distinctive feature of the considered boundary value problem is the inclusion of the Navier slip condition at the lower (solid) boundary of the layer instead of the classical condition of fluid no-slip on the solid surface. Modeling of the properties of the described flow is carried out using the system of Oberbeck-Boussinesq equations, its exact solution being obtained. The exact solution belongs to the Ostroumov-Birikh class. Hydrodynamic fields are described by polynomials. The degree of the polynomial describing the background pressure relative to the vertical coordinate is eight. The horizontal (longitudinal) pressure gradients are parabolic functions. This solution describes the multiple stratification of the pressure field. For the Navier slip condition and for special cases of no-slip and perfect slip conditions, corresponding studies are carried out to determine the number of pressure field stratification zones along the vertical coordinate.

Keywords: layered flow, Oberbeck-Boussinesq system of equations, exact solution, OstroumovBirikh class, unidirectional flow, counterflows, Marangoni convection, Navier condition.

\section{References}

1. Gershuni G.Z., Zhukhovitskii E.M. Convective Stability of Incompressible Fluids: Israel Program for Scientific Translations. Jerusalem, Keter Publishing House, 1976, 330 p.

2. $\quad$ Landau L.D., Lifshitz E.M. Fluid Mechanics, Pergamon Press, Oxford, 1987, 539 p.

3. Aristov S.N., Polyanin A.D. Exact solutions of unsteady three-dimensional navier-stokes equations. Doklady Physics, 2009, vol. 54, no. 7, pp. 316-321. DOI: 10.1134/S1028335809070039.

4. Polyanin A.D., Aristov S.N. Systems of hydrodynamic type equations: Exact solutions, transformations, and nonlinear stability. Doklady Physics, 2009, vol. 54, no. 9, pp. 429-434. DOI: $10.1134 /$ S1028335809090079.

5. Aristov S.N., Knyazev D.V., Polyanin A.D. Exact solutions of the Navier-Stokes equations with the linear dependence of velocity components on two space variables. Theoretical Foundations of Chemical Engineering, 2009, vol. 43, no. 5, pp. 642-662. DOI: 10.1134/S0040579509050066.

6. Aristov S.N., Pukhnachev V.V. On the Equations of Axisymmetric Motion of a Viscous Incompressible Fluid. Doklady Physics, 2004, vol. 49, no. 2, pp. 112-115. DOI: 10.1134/1.1686882. 
7. Aristov S.N., Prosviryakov E.Yu. A new class of exact solutions for three-dimensional thermal diffusion equations. Theoretical Foundations of Chemical Engineering, 2016, vol. 50, no. 3, pp. 286-293. DOI: 10.1134/S0040579516030027.

8. Khan Imad, Hussain Arif, Malik Muhammad Yousaf, Mukhtar Safyan. On magnetohydrodynamics Prandtl fluid flow in the presence of stratification and heat generation. Physica A: Statistical Mechanics and its Applications, 2019, vol. 540. DOI: 10.1016/j.physa.2019.123008.

9. Gopal D., Hari Shing Naik S., Kishan N., Raju C.S.K. The impact of thermal stratification and heat generation/absorption on MHD carreau nano fluid flow over a permeable cylinder. SN Applied Sciences, 2020, vol. 2, pp. 639. DOI: 10.1007/s42452-020-2445-5.

10. Khan Mair, Malik Muhammad Yousaf, Salahuddin T., Hussain Arif. Change in viscosity of Maxwell fluid flow due to thermal and solutal stratifications. Journal of Molecular Liquids, 2019, vol. 288, pp. 110970. DOI: 10.1016/j.molliq.2019.110970.

11. Knyazev D.V., Kolpakov I.Y. The exact solutions of the problem of a viscous fluid flow in a cylindrical domain with varying radius. Rus. J. Nonlin. Dyn., 2015, vol. 11, no. 1, pp. 89-97. DOI: 10.20537/nd1501004. (In Russian).

12. Borzenko E.I., Diakova O.A., Shrager G.R. Studying the slip phenomenon for a viscous fluid flow in a curved channel. Tomsk State University Journal of Mathematics and Mechanics, 2014, no. 2 (28), pp. 35-44. (In Russian).

13. Aristov S.N., Knyazev D.V. Three-dimensional viscous jet flow with plane free boundaries. Fluid Dynamics, 2017, vol. 52, no. 2, pp. 215-218. DOI: 10.1134/S0015462817020053.

14. Birikh R.V., Pukhnachev V.V., Frolovskaya O.A. Convective flow in a horizontal channel with non-Newtonian surface rheology under time-dependent longitudinal temperature gradient. Fluid Dyn., 2015, vol. 50, no. 1, pp. 173-179. DOI: 10.1134/S0015462815010172.

15. Garifullin F.A. Free convection in horizontal liquid layers. Soros Educational Journal, 2000, vol. 6, no. 8, pp.108-114. (In Russian).

16. Burmasheva N.V., Prosviryakov E.Yu. An exact solution to the description of a unidirectional Marangoni flow of a viscous incompressible fluid with the Navier boundary condition. Velocity field investigation. Diagnostics, Resource and Mechanics of materials and structure, 2019, iss. 5, pp. 23-39. DOI: 10.17804/2410-9908.2019.5.023-039. Available at: https://www.dream-journal.org/issues/2019-5/2019-5_259.html

17. Burmasheva N.V., Prosviryakov E.Yu. An exact solution for describing the unidirectional Marangoni flow of a viscous incompressible fluid with the Navier boundary condition. Temperature field investigation. Diagnostics, Resource and Mechanics of materials and structures, 2020, iss. 1, pp. 6-23. DOI: 10.17804/2410-9908.2020.1.006-023. Available at: https://www.dreamjournal.org/issues/2020-1/2020-1_278.html

18. Naresh Kumar Nedunuri, Ojjela Odelu, Sastry D.R.V.S.R.K. Effects of double stratification on MHD chemically reacting second-grade fluid through porous medium between two parallel plates. Heat Transfer-Asian Research, 2019, vol. 48, no. 8. DOI: 10.1002/htj.21564.

19. Abdul Wahab Hafiz, Zeb Hussan, Bhatti Sara, Nam Yunyoung. Numerical Study for the Effects of Temperature Dependent Viscosity Flow of Non-Newtonian Fluid with Double Stratification. Applied Sciences, 2020, vol. 10, no. 2, pp. 708. DOI: 10.3390/app10020708.

20. Burmasheva N.V., Prosviryakov E.Yu. Thermocapillary Convection of a Vertical Swirling Liquid. Theoretical Foundations of Chemical Engineering, 2020, vol. 54, no. 1, pp. 230-239. DOI: 10.1134/S0040579519060034.

21. Burmasheva N.V., Larina E.A., Prosviryakov E.Yu. Unidirectional Convective Flows of a Viscous Incompressible Fluid with Slippage in a Closed Layer. In: AIP Conference Proceedings, 2019, vol. 2176, pp. 030023-1-03023-5. DOI: 10.1063/1.5135147.

22. Mosina E.V., Chernyshev I.V. The permeability of two dimentional porous medium of square fibers (cell model). Science Journal of Volgograd State University. Mathematics. Physics, 2017, no. 2 (39), pp. 56-64. DOI: 10.15688/jvolsu1.2017.2.5. (In Russian). 
23. Gorshkov A.V., Prosviryakov E.Yu. Analytic solutions of stationary complex convection describing a shear stress field of different signs. Trudy IMM, 2017, vol. 23, no. 2, pp. 32-41. DOI: 10.21538/0134-4889-2017-23-2-32-41. (In Russian).

24. Burmasheva N.V., Prosviryakov E.Yu. Convective layered flows of a vertically whirling viscous incompressible fluid. Velocity field investigation. Vestnik Samarskogo Gosudarstvennogo Tekhnicheskogo Universiteta-Seriyafiziko-Matematicheskiye Nauki, 2019, vol. 23, no. 2, pp. 341-360. DOI: $10.14498 /$ vsgtu1670.

25. Privalova V.V., Prosviryakov E.Yu. Steady convective Coutte flow for quadratic heating of the lower boundary fluid layer. Russian Journal of Nonlinear Dynamics, 2018, vol. 14, no. 1, pp. 69-79. DOI: 10.20537/nd1801007.

26. Petrov A.G. Exact solution of the Navier-Stokes equations in a fluid layer between the moving parallel plates. Journal of Applied Mechanics and Technical Physics, 2012, vol. 53, no. 5, pp. 642-646. DOI: 10.1134/S0021894412050021.

27. Kolchanov N.V., Putin G.F. Gravitational convection of magnetic colloid in a horizontal layer. International Journal of Heat and Mass Transfer, 2015, vol. 89, pp. 90-101. DOI: 10.1016/j.ijheatmasstransfer.2015.05.050.

28. Burmasheva N.V., Prosviryakov E.Yu. Exact solution of the Navier-Stokes equations describing spatially inhomogeneous flows of a rotating fluid. Trudy Instituta matematiki $i$ mekhaniki UrO RAN, 2020, vol. 26, no. 2 (In Russian). (In print).

29. Gorshkov A.V., Prosviryakov E.Y. Ekman Convective Layer Flow of a Viscous Incompressible Fluid. Izvestiya. Atmospheric and Oceanic Physics, 2018, vol. 54, no. 2, pp. 189-195. DOI: $10.1134 /$ S0001433818020081.

30. Privalova V.V., Prosviryakov E.Yu., Simonov M.A. Nonlinear gradient flow of a vertical vortex fluid in a thin layer. Russian Journal of Nonlinear Dynamics, 2019, vol. 15, no. 3, 271-283. DOI: $10.20537 / \mathrm{nd} 190306$.

31. Navier C.L.M.H. M'emoire sur les lois du mouvement des fluids. M'em. Acad. Sci. Inst. de France, 1823, vol. 2, no. 6. pp. 389-440.

32. Ostroumov G.A. Free convection under the condition of the internal problem. Washington, NACA Technical Memorandum 1407, National Advisory Committee for Aeronautics, 1958.

33. Birikh R.V. Thermocapillary convection in a horizontal layer of liquid. J. Appl. Mech. Tech. Phys., 1966, no. 7, pp. 43-44. DOI: 10.1007/BF00914697.

34. Marangoni C. Sull espansione delle goccie di un liquido galleggiante sulla superficie di altro liquid, Pavia, Tipografia dei fratelli Fusi, 1865. 
Подана в журнал: 07.03.2020

УДК 539

DOI: $10.17804 / 2410-9908.2020 .2 .061-075$

\title{
ТОЧНОЕ РЕШЕНИЕ ДЛЯ ОПИСАНИЯ ОДНОНАПРАВЛЕННОГО ТЕЧЕНИЯ МАРАНГОНИ ВЯЗКОЙ НЕСЖИМАЕМОЙ ЖИДКОСТИ С ГРАНИЧНЫМ УСЛОВИЕМ НАВЬЕ: ИССЛЕДОВАНИЕ ПОЛЯ ДАВЛЕНИЯ
}

\author{
Н. В. Бурмашева ${ }^{\text {a)* }}$ Е. Ю. Просвиряков ${ }^{\text {() }}$ \\ Институт машиноведения УрО РАН, \\ ул. Комсомольская, 34, г. Екатеринбург, Российская Федерация \\ a) (iD https://orcid.org/0000-0003-4711-1894 nat_burm@mail.ru; \\ б) (iD https://orcid.org/0000-0002-2349-7801 《evgen_pros@mail.ru \\ *Ответственный автор. Электронная почта: nat_burm@mail.ru \\ Адрес для переписки: ул. Комсомольская, 34, г. Екатеринбург, Российская Федерация \\ Тел.: +7 (343) 375-35-76; факс: 374-53-30
}

В статье исследуются свойства поля давления при однонаправленном термокапиллярном течении вязкой несжимаемой жидкости в бесконечном горизонтальном слое заданной толщины. Отличительной особенностью рассматриваемой краевой задачи является учет условия проскальзывания Навье на нижней (твердой) границе слоя вместо классического условия прилипания жидкости к твердой поверхности. При моделировании свойств описанного течения при помощи системы уравнений Обербека-Буссинеска получено ее точное решение, принадлежащее классу Остроумова-Бириха. Гидродинамические поля описываются многочленами. Степень многочлена, описывающего фоновое давление, относительно вертикальной координаты равняется восьми. Горизонтальные (продольные) градиенты давления являются параболическими функциями. Данное решение описывает множественную стратификацию поля давления. Для условия скольжения Навье и для частных случаев условия прилипания и идеального скольжения проведены соответствующие исследования по определению количества зон стратификации поля давления по вертикальной координате.

Ключевые слова: слоистое течение, система уравнений Обербека-Буссинеска, точное решение, класс Остроумова-Бириха, однонаправленное течение, противотечения, конвекция Марангони, условие Навье.

\section{1. Введение}

Давление является одним из параметров, входящих в уравнение движения вязкой жидкости (уравнение Навье-Стокса) [1, 2], а следовательно, влияющих на свойства потока этой жидкости. Однако связь между скоростями течения и давлением, определяемую уравнением движения, можно рассматривать и в обратном контексте: каким образом характеристики течения влияют на топологию поля давления [3-6].

Известно, что в полную систему уравнений, описывающих конвективное течение вязкой жидкости, также входят уравнение теплопроводности и закон сохранения массы [1]. Эти три уравнения составляют замкнутую систему уравнений относительно вектора скоростей течения, температуры и давления. При этом все неизвестные функции (за исключением давления) входят, по крайней мере, в два из перечисленных трех уравнений. Это приводит к тому, что фактически поле давления определяется по уже известным значениям скорости течения и температуры. Таким образом, на свойства поля давления оказывают влияние особенности распределения поля скорости и температурного поля [7-15]. 
В $[16,17]$ было показано, что при определенном сочетании граничных условий возможно возникновение множественной стратификации поля скорости и температуры [8-10; 18-21]. И число точек стратификации этих полей зависит от конкретных значений управляющих параметров краевой задачи $[11,12,22-25]$. В данной статье исследуется влияние параметров граничных условий на распределение изолиний поля давления в горизонтальном слое [13-15; 26-30] при учете условия проскальзывания Навье [31] на нижней границе рассматриваемого слоя.

\section{2. Постановка задачи и методы решения}

Рассматривается установившееся однонаправленное течение вязкой несжимаемой жидкости в горизонтальном слое заданной толщины $h$ (рис. 1). Полагаем, что движение происходит в поле силы тяжести с ускорением свободного падения $\boldsymbol{g}$. Кроме того, принимаем приближение Обербека-Буссинеска о влиянии температуры жидкости $T$ на ее плотность $\rho=\rho_{0}(1-\beta T)$, здесь $\beta$ - коэффициент объемного расширения жидкости $[1,2]$.

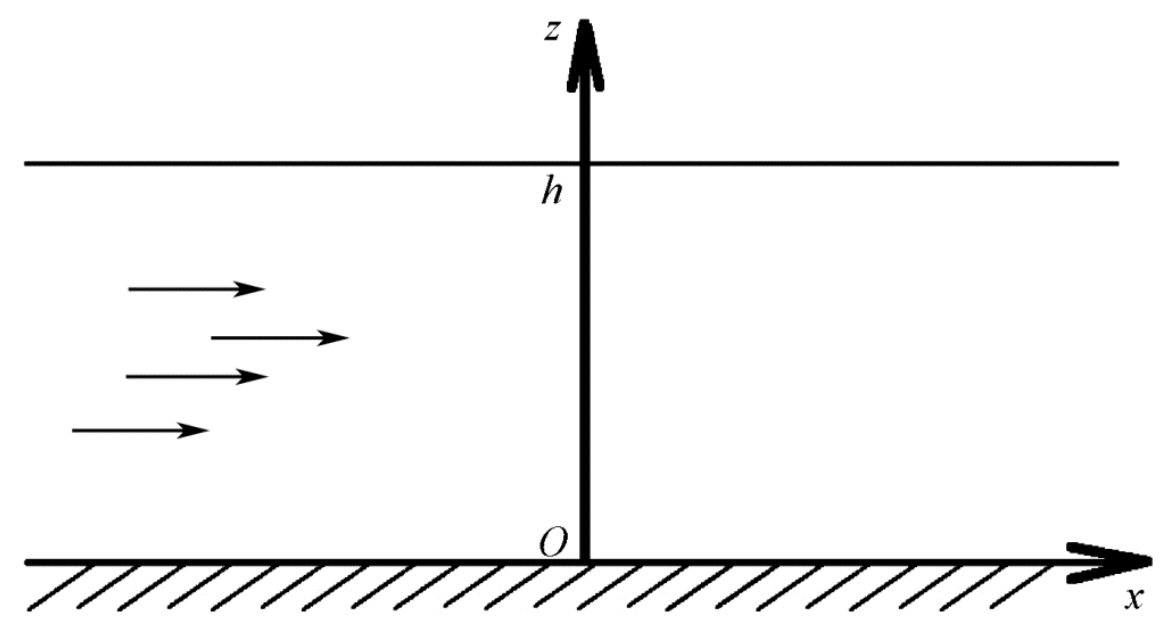

Рис. 1. Геометрия слоя жидкости

Для однонаправленного течения вдоль оси $O x$ базовая система уравнений тепловой конвекции принимает вид $[16,17,20,21,23-25 ; 28-30]$ :

$$
\begin{gathered}
V_{x} \frac{\partial V_{x}}{\partial x}=-\frac{\partial P}{\partial x}+v\left(\frac{\partial^{2} V_{x}}{\partial x^{2}}+\frac{\partial^{2} V_{x}}{\partial y^{2}}+\frac{\partial^{2} V_{x}}{\partial z^{2}}\right) ; \\
\frac{\partial P}{\partial y}=0 ; \frac{\partial P}{\partial z}=g \beta T ; \\
V_{x} \frac{\partial T}{\partial x}=\chi\left(\frac{\partial^{2} T}{\partial x^{2}}+\frac{\partial^{2} T}{\partial y^{2}}+\frac{\partial^{2} T}{\partial z^{2}}\right) ; \\
\frac{\partial V_{x}}{\partial x}=0
\end{gathered}
$$

Здесь $V_{x}(x, y, z)$ - скорость течения вдоль выбранного направления; $P(x, y, z)$ - нормированное на плотность отклонение давления от гидростатического; $T(x, y, z)$ - отклонение температуры от отсчетного значения; $v, \chi$ - кинематическая (молекулярная) вязкость и температуропроводность жидкости соответственно. В роли неизвестных в системе (1) выступа- 
ют три функции - давление $P$, температура $T$ и скорость $V_{x}$. Отметим, что их число меньше числа уравнений системы (1), т. е. речь идет об исследовании переопределенной системы.

В [16] было исследовано поле скорости течения, профиль которой зависит только от одной (поперечной) координаты, т. е. скорость представима в следующем виде:

$$
V_{x}=U(z)
$$

Также в [16] было показано, что если скорость течения имеет структуру (2), то вид решения для поля температуры и поля давления есть двумерные по координатам функции следующего вида:

$$
T=T_{0}(z)+T_{1}(z) x ; P=P_{0}(z)+P_{1}(z) x .
$$

Точное решение (2) и (3) обобщается в известное семейство Остроумова-Бириха $[7,16,17,20,21,24,28,32,33]$.

Структура выражений (2), (3) позволяет преобразовать систему уравнений (1) в частных производных к системе обыкновенных дифференциальных уравнений:

$$
\begin{gathered}
T_{1}^{\prime \prime}=0 ; P_{1}^{\prime}=g \beta T_{1} ; \nu U^{\prime \prime}=P_{1} ; \\
\chi T_{0}^{\prime \prime}=U T_{1} ; P_{0}^{\prime}=g \beta T_{0} .
\end{gathered}
$$

Здесь штрихом обозначено дифференцирование по поперечной (вертикальной) переменной $z$. Общее решение системы (4) для поля давления имеет вид:

$$
\begin{gathered}
P_{1}(z)=\frac{1}{2} g z^{2} \beta C_{1}+g z \beta C_{2}+C_{3} ; \\
P_{0}(z)=\frac{g^{2} z^{8} \beta^{2} C_{1}^{2}}{8064 v \chi}+\frac{g^{2} z^{7} \beta^{2} C_{1} C_{2}}{1008 v \chi}+\frac{g z^{6} \beta\left[g \beta C_{2}^{2}+3 C_{1} C_{3}\right]}{720 v \chi}+\frac{g z^{5} \beta\left[C_{2} C_{3}+2 v C_{1} C_{4}\right]}{120 v \chi}+ \\
+\frac{g z^{4} \beta\left(C_{2} C_{4}+C_{1} C_{5}\right)}{24 \chi}+\frac{g z^{3} \beta C_{2} C_{5}}{6 \chi}+\frac{1}{2} g z^{2} \beta C_{6}+g z \beta C_{7}+C_{8} .
\end{gathered}
$$

Также в [16] сформулированы восемь краевых условий для определения значений восьми неизвестных постоянных интегрирования $C_{1}, \ldots, C_{8}$. Эти краевые условия описывают возникновение конвекции Марангони [33, 34] вследствие задания неоднородного источника тепла на верхней границе слоя и учета условия проскальзывания Навье [31] на нижней границе слоя. Математически в силу структуры решений (2) и (3) эти условия записываются в виде следующих соотношений:

$$
\begin{gathered}
T_{0}(0)=T_{1}(0)=0 ; T_{0}(h)=0 ; T_{1}(h)=A ; \\
\left.\alpha \frac{\partial U}{\partial z}\right|_{z=0}=U(0) ; P_{0}(h)=S_{0} ; P_{1}(h)=0 ;\left.\eta \frac{\partial U}{\partial z}\right|_{z=h}=-\sigma T_{1}(h) .
\end{gathered}
$$

Здесь $\sigma$ и $\eta$ - коэффициенты температурного поверхностного натяжения и динамической вязкости жидкости соответственно; $\alpha$ - длина проскальзывания. 


\section{3. Результаты и обсуждение}

\section{1. Точное решение краевой задачи}

Частное решение системы (4) для компонент поля давления, отвечающее граничным условиям (6), представляет собой набор полиномиальных функций следующего вида:

$$
\begin{gathered}
P_{1}=\frac{A g \beta}{2 h}\left(z^{2}-h^{2}\right) ; \\
P_{0}=S_{0}-\frac{A^{2} g \beta \sigma}{120 h \eta \chi}(z-h)^{2}\left(3 h^{3}+2 h z(2 z+5 \alpha)+z^{2}(2 z+5 \alpha)+h^{2}(6 z+5 \alpha)\right)+ \\
+\frac{A^{2} g^{2} \beta^{2}}{40320 h^{2} v \chi}(z-h)^{2}\left[183 h^{6}-69 h^{2} z^{4}+10 h z^{5}+5 z^{6}+\right. \\
\left.+h^{4} z(221 z+1120 \alpha)+h^{5}(366 z+560 \alpha)+4 h^{3} z^{2}(19 z+140 \alpha)\right] .
\end{gathered}
$$

Ранее были достаточно подробно проанализированы точные решения (7) для поля скорости $\boldsymbol{V}$ [16] и поля температуры $T$ [17]. В статье будет проведено исследование свойств точного решения (7), описывающего поле давления $P$.

Из решения (7) следует, что если продольный градиент температуры $A=0$, то распределение поля давления $P$ оказывается однородным:

$$
\left.P\right|_{A=0}=\left.\left(P_{0}(z)+P_{1}(z) x\right)\right|_{A=0}=S_{0} .
$$

Расслоений поля давления при таком задании не возникает, поэтому всюду далее будем полагать, что горизонтальный градиент температуры $A$ отличен от нуля.

Заметим, что продольный градиент давления $P_{1}$ в полученном выражении (7) не зависит от длины проскальзывания $\alpha$ и при любом сочетании значений параметров $A, \beta, h$ не обращается в нуль внутри слоя $(0, h)$, следовательно, отвечающее этому градиенту поле $P_{1} x$ не имеет точек стратификации.

\section{2. Исследование поля давления в случае прилипания жидкости}

В случае выполнения условия прилипания жидкости на твердой границе решение (7) для поля давления можно переписать в виде:

$$
\begin{gathered}
P_{1}=\frac{A g \beta h}{2}\left(Z^{2}-1\right) ; \\
P_{0}=S_{0}-\frac{A^{2} g \beta \sigma h^{4}}{120 \eta \chi}(Z-1)^{2}\left(3+6 Z+4 Z^{2}+2 Z^{3}\right)+ \\
+\frac{A^{2} g^{2} \beta^{2} h^{6}}{40320 v \chi}(Z-1)^{2}\left[183+366 Z+221 Z^{2}+76 Z^{3}-69 Z^{4}+10 Z^{5}+5 Z^{6}\right]= \\
=S_{0}-\frac{A^{2} g \beta \sigma h^{4}}{120 \eta \chi} f_{1}(Z)+\frac{A^{2} g^{2} \beta^{2} h^{6}}{40320 v \chi} f_{2}(Z) .
\end{gathered}
$$

В формулах (8) введена безразмерная координата $Z=z / h \in[0,1]$. Фоновое давление $P_{0}$ определяется взаимодействием постоянного однородного давления, термокапиллярного и термогравитационного слагаемых. При варьировании толщины слоя $h$ постоянное давление $S_{0}$ будет 
вносить превалирующий вклад в фоновое давление $P_{0}$. На рис. 2 представлены профили функций $f_{1}(Z)$ и $f_{2}(Z)$, входящих в состав выражения (8) для фонового давления.

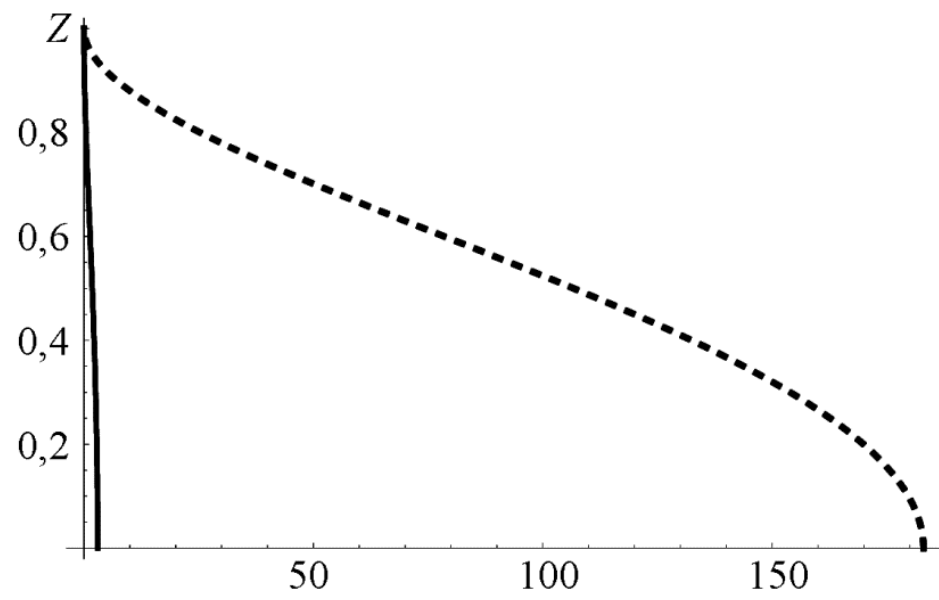

Рис. 2. Профили вспомогательных функций $f_{1}(Z)=(Z-1)^{2}\left(3+6 Z+4 Z^{2}+2 Z^{3}\right)$ (сплошная линия) и $f_{2}(Z)=(Z-1)^{2}\left[183+366 Z+221 Z^{2}+76 Z^{3}-69 Z^{4}+10 Z^{5}+5 Z^{6}\right]$ (пунктирная линия)

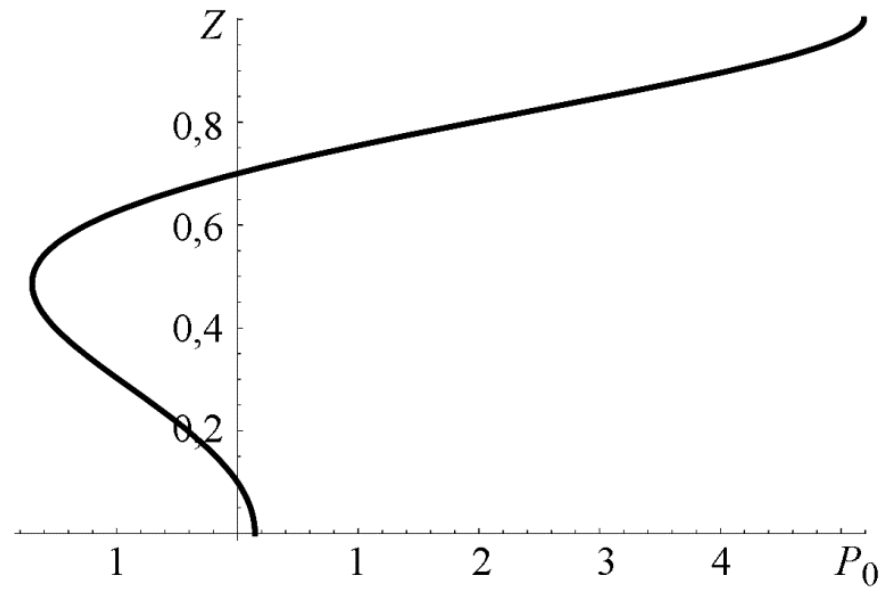

Рис. 3. Профиль фонового давления $P_{0}$ в случае прилипания жидкости:

$$
\text { при } S_{0}=5,188 \mathrm{H} / \mathrm{m}^{2} ; \frac{A^{2} g \beta \sigma h^{4}}{120 \eta \chi}=62,681 \mathrm{H} / \mathrm{m}^{2} ; \frac{A^{2} g^{2} \beta^{2} h^{6}}{40320 v \chi}=1 \mathrm{H} / \mathrm{m}^{2}
$$

Поскольку функции $f_{1}(Z)$ и $f_{2}(Z)$ принимают строго положительные значения внутри рассматриваемого слоя, а параметр $S_{0}$ отвечает атмосферному давлению, на основании структуры выражения (8) можно сделать вывод, что фоновое давление $P_{0}$ не может иметь нулевых точек внутри слоя, если жидкость является аномальной $(\sigma<0)$. Другими словами, стратификация поля $P_{0}$ возможна только для ньютоновских жидкостей.

Функции $f_{1}(Z)$ и $f_{2}(Z)$ строго монотонны на отрезке [0,1], а значит, по аналогии с подходом, использованным в [16] при оценке числа застойных точек поля скорости течения, можно утверждать, что число нулевых точек поля давления $P_{0}$ не превосходит двух. Профиль фонового давления для этого случая представлен на рис. 3 . 
Заметим, что картина распределения линий уровня поля давления $P$ при наличии двух нулевых точек у фонового давления $P_{0}$ зависит помимо прочего от величины коэффициента $A g \beta h / 2$, определяющего продольный градиент давления $P_{1}$ (рис. 4 и 5).

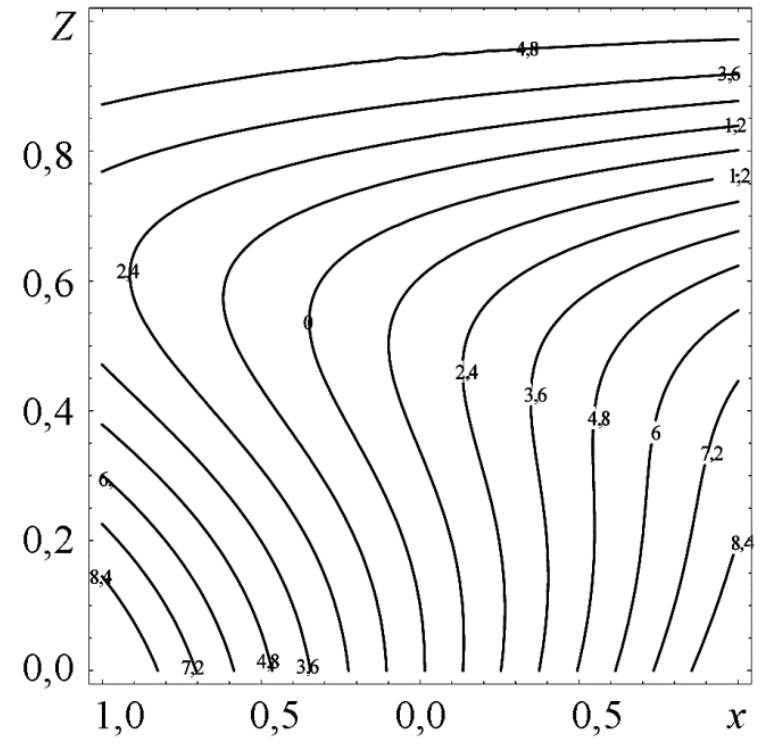

Рис. 4. Линии уровня поля давления в случае прилипания:

$$
\text { при } \begin{gathered}
S_{0}=5,188 ; \frac{A^{2} g \beta \sigma h^{4}}{120 \eta \chi}=62,681 ; \\
\frac{A^{2} g^{2} \beta^{2} h^{6}}{40320 v \chi}=1 ; \frac{A g \beta h}{2}=10
\end{gathered}
$$

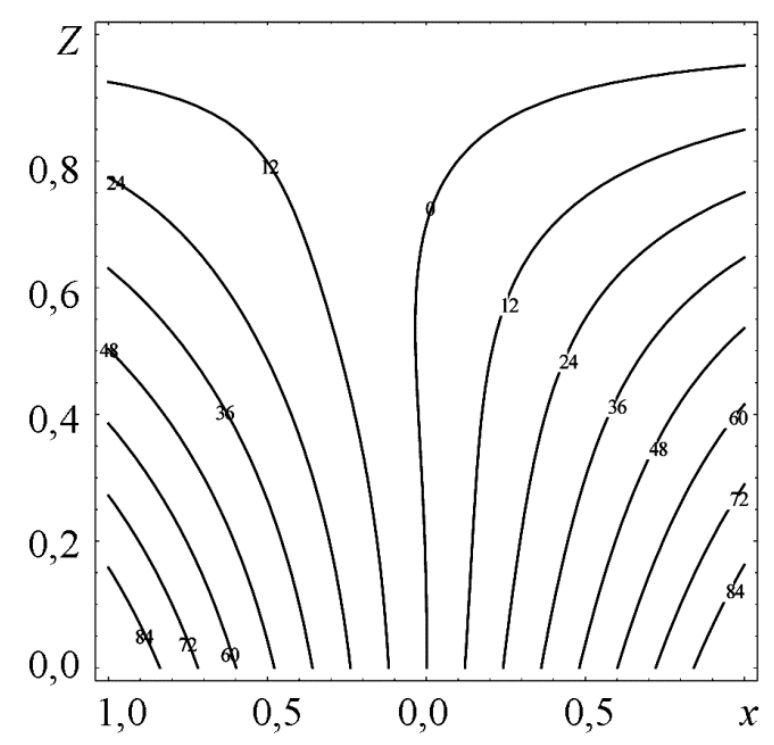

Рис. 5. Линии уровня поля давления в случае прилипания:

$$
\text { при } \begin{gathered}
S_{0}=5,188 ; \frac{A^{2} g \beta \sigma h^{4}}{120 \eta \chi}=62,681 ; \\
\frac{A^{2} g^{2} \beta^{2} h^{6}}{40320 v \chi}=1 ; \frac{A g \beta h}{2}=100
\end{gathered}
$$

Рис. 4 и 5 наглядно демонстрируют, что число нулевых точек поля давления меняется в зависимости от удаленности выбранного среза от сечения $x=0$.

\section{3. Исследование поля давления в случае проскальзывания жидкости}

Рассмотрим теперь, какой вклад вносят слагаемые в выражении (7), содержащие длину скольжения $\alpha$. Для этого представим точное решение (7) в следующем виде:

$$
\begin{gathered}
P_{0}=S_{0}-\frac{A^{2} g \beta \sigma h^{4}}{120 \eta \chi}(Z-1)^{2}\left(3+6 Z+4 Z^{2}+2 Z^{3}\right)+ \\
+\frac{A^{2} g^{2} \beta^{2} h^{6}}{40320 v \chi}(Z-1)^{2}\left[183+366 Z+221 Z^{2}+76 Z^{3}-69 Z^{4}+10 Z^{5}+5 Z^{6}\right]+ \\
+\left(-5 \frac{A^{2} g \beta \sigma h^{3}}{120 \eta \chi}+560 \frac{A^{2} g^{2} \beta^{2} h^{2}}{40320 v \chi}\right)\left(Z^{2}-1\right)^{2} \alpha .
\end{gathered}
$$

Отметим, что в отличие от анализа выражения (8) сказать что-либо о природе жидкости (ньютоновская/аномальная) на основе решения (9) уже не представляется возможным. 
Кроме того, коэффициент $\left(-5 \frac{A^{2} g \beta \sigma h^{3}}{120 \eta \chi}+560 \frac{A^{2} g^{2} \beta^{2} h^{2}}{40320 v \chi}\right)$ при дополнительном слагаемом в выражении (9) оказался связан с коэффициентами $\frac{A^{2} g \beta \sigma h^{3}}{120 \eta \chi}$ и $\frac{A^{2} g^{2} \beta^{2} h^{2}}{40320 v \chi}$, стоящими перед функциями $f_{1}(Z)$ и $f_{2}(Z)$ в точных решениях (8) и (9). Поэтому, несмотря на появление дополнительного независимого положительного параметра $\alpha$, число нулевых точек многочлена (9) также не превосходит двух. Соответствующий профиль приведен на рис. 6.

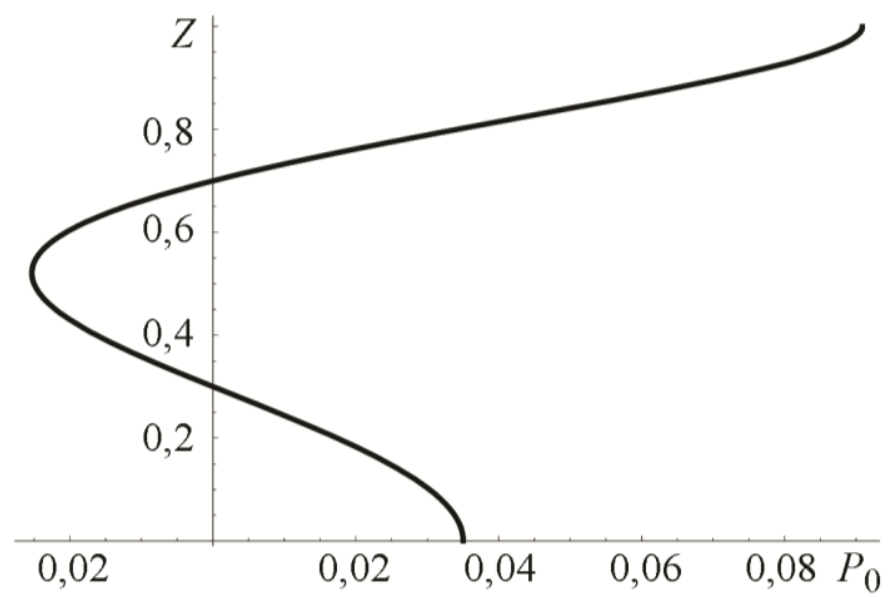

Рис. 6. Профиль фонового давления $P_{0}$ в случае выполнения условия Навье:

при $S_{0}=0,00097 \mathrm{H} / \mathrm{m}^{2} ; \frac{A^{2} g \beta \sigma h^{4}}{120 \eta \chi}=1 \mathrm{H} / \mathrm{m}^{2} ; \frac{A^{2} g^{2} \beta^{2} h^{6}}{40320 v \chi}=0,01069 \mathrm{H} / \mathrm{m}^{2} ; \alpha=1 \mathrm{м}$

\section{4. Исследование поля давления в случае идеального скольжения}

Если длина скольжения $\alpha=\infty$, то условие Навье изменит вид - вместо выражения

$$
\left.\alpha \frac{\partial U}{\partial z}\right|_{z=0}=U(0)
$$

входящего в группу краевых условий (6), получим (в силу предельного перехода) условие следующего вида:

$$
\left.\frac{\partial U}{\partial z}\right|_{z=0}=0
$$

В [17] было получено точное решение для фоновой температуры $T_{0}$ в случае идеального скольжения:

$$
T_{0}=\frac{A^{2} g \beta h^{3}}{5040 v \chi}\left[5 Z^{7}-63 Z^{5}+63 Z^{3}-5 Z\right]
$$


Подставим далее выражение (11) в последнее уравнение системы (4)

$$
P_{0}^{\prime}=g \beta T_{0}=\frac{A^{2} g^{2} \beta^{2} h^{3}}{5040 v \chi}\left[5 Z^{7}-63 Z^{5}+63 Z^{3}-5 Z\right]
$$

и проинтегрируем его. В результате получим точное решение для фонового давления $P_{0}$ :

$$
\begin{aligned}
P_{0} & =\frac{A^{2} g^{2} \beta^{2} h^{3}}{5040 v \chi}\left[\frac{5}{8} Z^{8}-\frac{63}{6} Z^{6}+\frac{63}{4} Z^{4}-\frac{5}{2} Z^{2}\right]+C_{1}= \\
& =\frac{A^{2} g^{2} \beta^{2} h^{3}}{40320 v \chi}\left[5 Z^{8}-84 Z^{6}+126 Z^{4}-20 Z^{2}\right]+C_{1} .
\end{aligned}
$$

Для определения константы $C_{1}$ воспользуемся граничным условием $P_{0}(z=h)=S_{0}$, которое в результате замены $z$ на $Z$ примет вид:

$$
P_{0}(Z=1)=S_{0}
$$

Подставив в это условие решение (12), получим:

$$
S_{0}=\frac{A^{2} g^{2} \beta^{2} h^{3}}{40320 v \chi}[5-84+126-20]+C_{1}=\frac{27 A^{2} g^{2} \beta^{2} h^{3}}{40320 v \chi}+C_{1},
$$

откуда находим, что $C_{1}=S_{0}-\frac{27 A^{2} g^{2} \beta^{2} h^{3}}{40320 v \chi}$. Следовательно, выражение для фонового давления в случае идеального скольжения жидкости имеет вид:

$$
P_{0}=S_{0}-\frac{A^{2} g^{2} \beta^{2} h^{3}}{40320 v \chi}\left[5 Z^{8}-84 Z^{6}+126 Z^{4}-20 Z^{2}-27\right]
$$

Отметим, что функция $g(Z)=5 Z^{8}-84 Z^{6}+126 Z^{4}-20 Z^{2}-27$, входящая в выражение (13), не имеет нулей на интервале $(0,1)$, но при этом не является монотонной (имеет на данном интервале одну точку экстремума - точку $Z_{g}=0,2947155529756107$ ) (рис. 7).

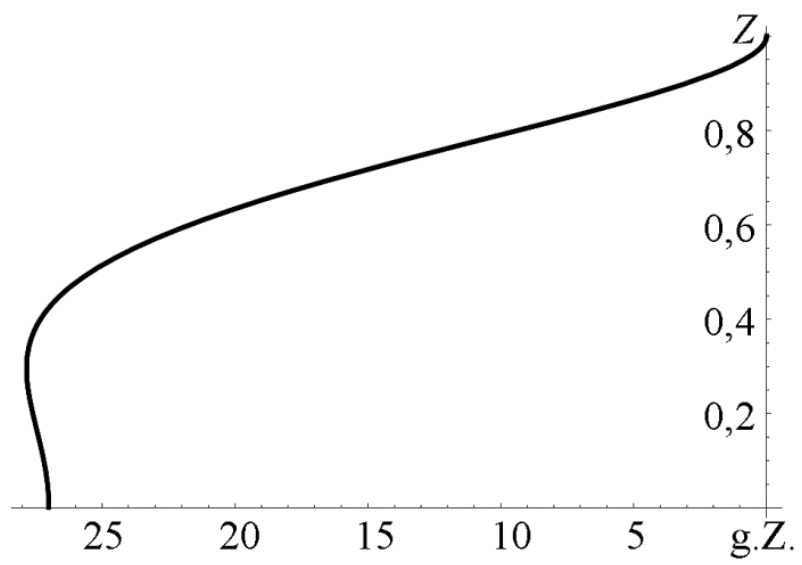

Рис. 7. Профиль функции $g(Z)$ 
Следовательно, у фонового давления (13) могут быть две нулевые точки внутри рассматриваемого слоя $(0,1)$, причем обе эти точки находятся в окрестности точки $Z_{g}$ (рис. 8 ).

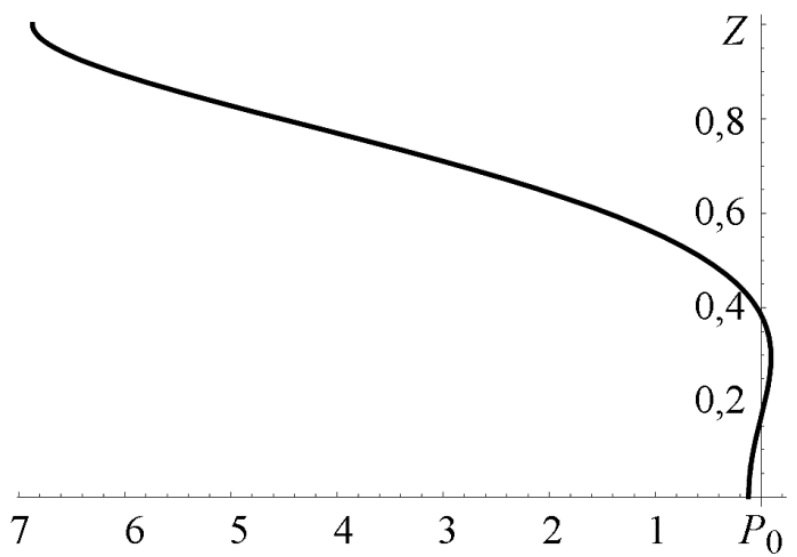

Рис. 8. Профиль фонового давления $P_{0}$ в случае идеального скольжения жидкости:

$$
\text { при } S_{0}=-6,87 ; \frac{A^{2} g^{2} \beta^{2} h^{3}}{40320 v \chi}=0,25
$$

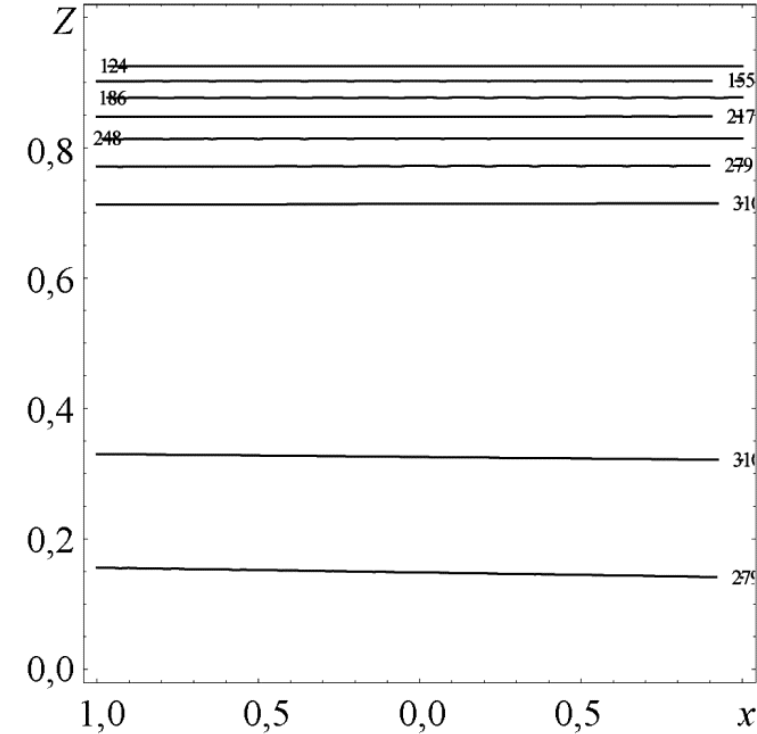

Рис. 9. Изолинии поля давления $P$ при $S_{0}=1 ; b_{1}=1 ; b_{2}=10$

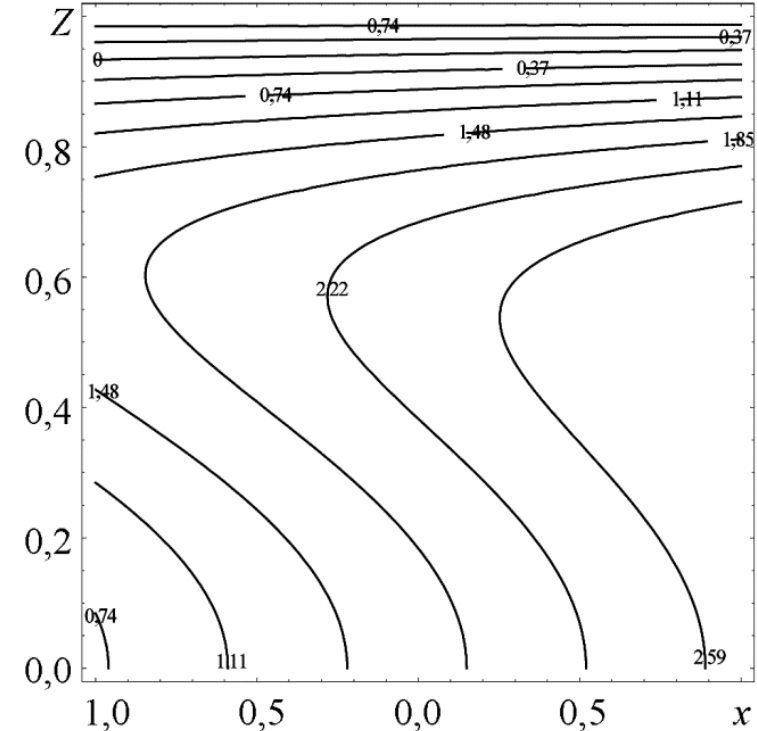

Рис. 10. Изолинии поля давления $P$ при $S_{0}=1 ; b_{1}=1 ; b_{2}=0,1$

Заметим, что стратификация фонового давления $P_{0}$ на три зоны возможная для любой жидкости. Используя соотношения (8) и (13), легко находим выражение для итогового поля давления $P$ :

$$
\begin{aligned}
P=P_{0}+P_{1} x & =S_{0}-\frac{A^{2} g^{2} \beta^{2} h^{3}}{40320 v \chi}\left[5 Z^{8}-84 Z^{6}+126 Z^{4}-20 Z^{2}-27\right]+\frac{A g \beta h}{2}\left(Z^{2}-1\right) x= \\
& =S_{0}-\frac{A^{2} g^{2} \beta^{2} h^{3}}{40320 v \chi}\left(Z^{2}-1\right)\left(5 Z^{4}-74 Z^{2}-27\right)+\frac{A g \beta h}{2}\left(Z^{2}-1\right) x=
\end{aligned}
$$




$$
=S_{0}-\frac{A g \beta h}{2}\left(Z^{2}-1\right)\left[\frac{A g \beta h^{2}}{20160 v \chi}\left(5 Z^{4}-74 Z^{2}-27\right)-x\right] .
$$

Из формулы (14) следует, что распределение изолиний поля давления зависит от величины параметра $S_{0}$ и коэффициентов $b_{1}=A g \beta h / 2, b_{2}=A g \beta h^{2} /(20160 v \chi)$ (рис. 9-11).

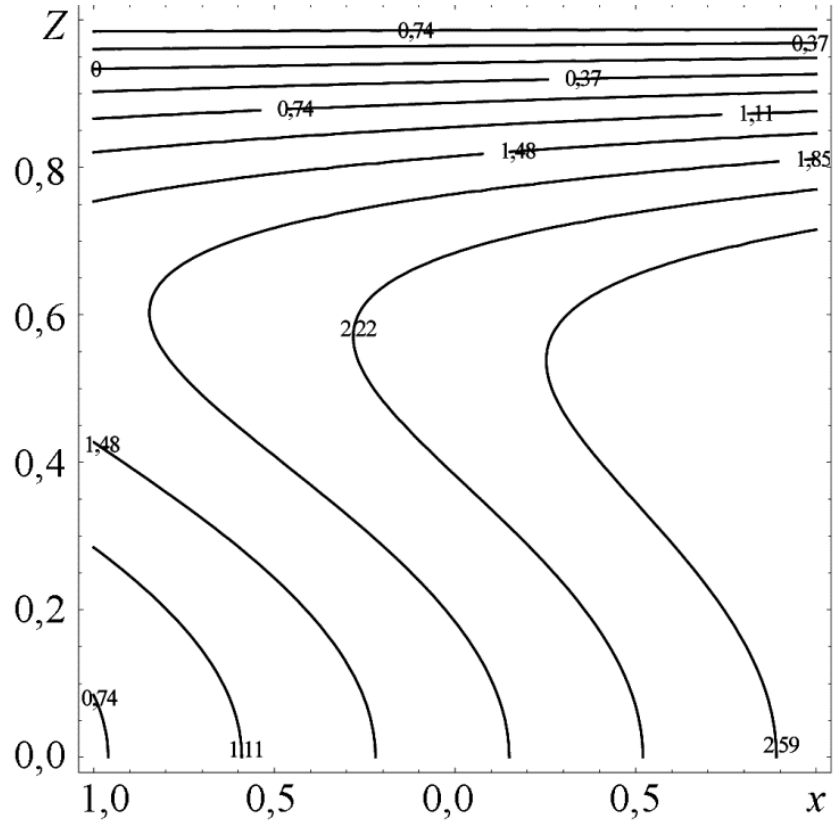

Рис. 11. Изолинии поля давления $P$ при $S_{0}=1 ; b_{1}=1 ; b_{2}=0,01$

Отметим, что изменение порядка величины коэффициентов $b_{1}, b_{2}$ возможно за счет выбора жидкости, за счет изменения параметра нагрева границ и за счет варьирования толщины слоя жидкости.

\section{4. Заключение}

В статье предложено точное решение системы уравнений Обербека-Буссинеска, описывающее однонаправленное течение вязкой несжимаемой жидкости в горизонтальном слое, индуцированное учетом термокапиллярного эффекта и условия проскальзывания Навье. Основное внимание в статье было уделено изучению свойств поля давления. Показано, что независимо от величины длины скольжения фоновая температура может стратифицироваться на три зоны относительно отсчетного значения. Однако положение точек стратификации зависит от физических свойств жидкости и от значений параметров, определяющих краевую задачу.

\section{Литература}

1. Гершуни Г. 3., Жуховицкий Е. М. Конвективная неустойчивость несжимаемой жидкости. - М. : Наука, 1972. - 532 с.

2. Ландау Л. Д., Лифшиц Е. М. Гидродинамика. - 6-е. изд. - М. : Физматлит, 2006. - 736 с. 3. Aristov S. N., Polyanin A. D. Exact solutions of unsteady three-dimensional navier-stokes equations // Doklady Physics. - 2009. - Vol. 54, no. 7. - P. 316-321. - DOI: 10.1134/S1028335809070039. 
4. Polyanin A. D., Aristov S. N. Systems of hydrodynamic type equations: Exact solutions, transformations, and nonlinear stability // Doklady Physics. - 2009. - Vol. 54, no. 9. - P. 429-434. DOI: $10.1134 / \mathrm{S} 1028335809090079$.

5. Aristov S. N., Knyazev D. V., Polyanin A. D. Exact solutions of the Navier-Stokes equations with the linear dependence of velocity components on two space variables // Theoretical Foundations of Chemical Engineering. - 2009. - Vol. 43, no. 5. - P. 642-662. DOI: $10.1134 / \mathrm{S} 0040579509050066$.

6. Aristov S. N., Pukhnachev V. V. On the Equations of Axisymmetric Motion of a Viscous Incompressible Fluid // Doklady Physics. - 2004. - Vol. 49, no. 2. - P. 112-115. DOI: $10.1134 / 1.1686882$.

7. Aristov S. N., Prosviryakov E. Yu. A new class of exact solutions for three-dimensional thermal diffusion equations // Theoretical Foundations of Chemical Engineering. - 2016. - Vol. 50, no. 3. - P. 286-293. - DOI: 10.1134/S0040579516030027.

8. On magnetohydrodynamics Prandtl fluid flow in the presence of stratification and heat generation / Imad Khan, Arif Hussain, Muhammad Yousaf Malik, Safyan Mukhtar // Physica A: Statistical Mechanics and its Applications. - 2019. - Vol. 540. - DOI: 10.1016/j.physa.2019.123008.

9. The impact of thermal stratification and heat generation/absorption on MHD carreau nano fluid flow over a permeable cylinder / D. Gopal, S. Hari Shing Naik, N. Kishan, C. S. K. Raju // SN Applied Sciences. - 2020. - Vol. 2. - P. 639. - DOI: 10.1007/s42452-020-2445-5.

10. Change in viscosity of Maxwell fluid flow due to thermal and solutal stratifications / Mair Khan, Muhammad Yousaf Malik, T. Salahuddin, Arif Hussain // Journal of Molecular Liquids. 2019. - Vol. 288. - P. 110970. - DOI: 10.1016/j.molliq.2019.110970.

11. Князев Д. В., Колпаков И. Ю. Точные решения задачи о течении вязкой жидкости в цилиндрической области с меняющимся радиусом // Нелинейная динамика. - 2015. - Т. 11, № 1. - C. 89-97. - DOI: 10.20537/nd1501004.

12. Борзенко Е. И., Дьякова О. А., Шрагер Г. Р. Исследование явления проскальзывания в случае течения вязкой жидкости в изогнутом канале // Вестник Томского государственного университета. Математика и механика. - 2014. - № 2 (28). - С. 35-44.

13. Aristov S. N., Knyazev D. V. Three-dimensional viscous jet flow with plane free boundaries // Fluid Dynamics. - 2017. - Vol. 52, no. 2. - P. 215-218. - DOI: 10.1134/S0015462817020053.

14. Бирих Р. В., Пухначев В. В., Фроловская О. А. Конвективное течение в горизонтальном канале с неньютоновской реологией поверхности при нестационарном продольном градиенте температуры // Изв. РАН. Механика жидкости и газа. - 2015. - № 1. - С. 192-198.

15. Гарифуллин Ф. А. Возникновение конвекции в горизонтальных слоях жидкости // Соросовский образовательный журнал. - 2000. - Т. 6, № 8. - С.108-114.

16. Burmasheva N. V., Prosviryakov E. Yu. An exact solution to the description of a unidirectional Marangoni flow of a viscous incompressible fluid with the Navier boundary condition. Velocity field investigation // Diagnostics, Resource and Mechanics of materials and structures. 2019. - Iss. 5. - P. 23-39. - DOI: 10.17804/2410-9908.2019.5.023-039. - URL: https://www.dreamjournal.org/issues/2019-5/2019-5_259.html

17. Burmasheva N. V., Prosviryakov E. Yu. An exact solution for describing the unidirectional Marangoni flow of a viscous incompressible fluid with the Navier boundary condition. Temperature field investigation // Diagnostics, Resource and Mechanics of materials and structures. - 2020. Iss. 1. - P. 6-23. - DOI: 10.17804/2410-9908.2020.1.006-023. - URL: https://www.dreamjournal.org/issues/2020-1/2020-1_278.html

18. Nedunuri Naresh Kumar, Ojjela Odelu, Sastry D. R. V. S. R. K. Effects of double stratification on MHD chemically reacting second-grade fluid through porous medium between two parallel plates // Heat Transfer-Asian Research. - 2019. - Vol. 48, no. 8. - DOI: 10.1002/htj.21564. 
19. Numerical Study for the Effects of Temperature Dependent Viscosity Flow of Non-Newtonian Fluid with Double Stratification / Hafiz Abdul Wahab, Hussan Zeb, Sara Bhatti, Yunyoung Nam // Applied Sciences. - 2020. - Vol. 10, no. 2. - P. 708. - DOI: 10.3390/app10020708.

20. Burmasheva N. V., Prosviryakov E. Yu. Thermocapillary Convection of a Vertical Swirling Liquid // Theoretical Foundations of Chemical Engineering. - 2020. - Vol. 54, no. 1. - P. 230-239. DOI: $10.1134 / \mathrm{S} 0040579519060034$.

21. Burmasheva N. V., Larina E. A., Prosviryakov E. Yu. Unidirectional Convective Flows of a Viscous Incompressible Fluid with Slippage in a Closed Layer // AIP Conference Proceedings. 2019. - Vol. 2176. - P. 030023-1-03023-5. - DOI: 10.1063/1.5135147.

22. Мосина Е. В., Чернышев И. В. Проницаемость двумерной пористой среды из волокон квадратного сечения (ячеечная модель) // Вестник Волгоградского государственного университета. Серия 1. Математика. Физика. - 2017. - № 2 (39). - С. 56-64. - DOI: 10.15688/jvolsu1.2017.2.5.

23. Горшков А. В., Просвиряков Е. Ю. Аналитические решения стационарной сложной конвекции, описывающие поле касательных напряжений разного знака // Труды ИММ. 2017. - T. 23, № 2. - C. 32-41. - DOI: 10.21538/0134-4889-2017-23-2-32-41.

24. Burmasheva N. V., Prosviryakov E. Yu. Convective layered flows of a vertically whirling viscous incompressible fluid. Velocity field investigation // Vestnik Samarskogo Gosudarstvennogo Tekhnicheskogo Universiteta-Seriyafiziko-Matematicheskiye Nauki. - - 2019. - Vol. 23, no. 2. P. 341-360. - DOI: 10.14498/vsgtu1670.

25. Privalova V. V., Prosviryakov E. Yu. Steady convective Coutte flow for quadratic heating of the lower boundary fluid layer // Russian Journal of Nonlinear Dynamics. - 2018. - Vol. 14, no. 1. P. 69-79. - DOI: $10.20537 /$ nd1801007.

26. Petrov A. G. Exact solution of the Navier-Stokes equations in a fluid layer between the moving parallel plates // Journal of Applied Mechanics and Technical Physics. - 2012. - Vol. 53, no. 5. - P. 642-646. - DOI: 10.1134/S0021894412050021.

27. Kolchanov N. V., Putin G. F. Gravitational convection of magnetic colloid in a horizontal layer // International Journal of Heat and Mass Transfer. - 2015. - Vol. 89. - P. 90-101. DOI: 10.1016/j.ijheatmasstransfer.2015.05.050.

28. Бурмашева Н. В., Просвиряков Е. Ю. Точное решение уравнений Навье-Стокса, описывающее пространственно неоднородные течения вращающейся жидкости // Труды Института математики и механики УрО РАН. - 2020. - Т. 26, № 2. (Принято в печать).

29. Gorshkov A. V., Prosviryakov E. Y. Ekman Convective Layer Flow of a Viscous Incompressible Fluid // Izvestiya. Atmospheric and Oceanic Physics. - 2018. - Vol. 54, no. 2. - P. 189-195. DOI: $10.1134 / \mathrm{S} 0001433818020081$.

30. Privalova V. V., Prosviryakov E. Yu., Simonov M. A. Nonlinear gradient flow of a vertical vortex fluid in a thin layer // Russian Journal of Nonlinear Dynamics. - 2019. - Vol. 15, no. 3. P. 271-283. - DOI: https://doi.org/10.20537/nd190306.

31. Navier C. L. M. H. M'emoire sur les lois du mouvement des fluides // M'em. Acad. Sci. Inst. de France. - 1823. - Vol. 2, no. 6. - C. 389-440.

32. Остроумов Г. А. Свободная конвекция в условиях внутренней задачи. - М. : Гос. издво техн.-теорет. лит., 1952. - $256 \mathrm{c.}$

33. Birikh R. V. Thermocapillary convection in a horizontal layer of liquid. J. Appl. Mech. Tech. Phys., 1966, no. 7, pp. 43-44. - DOI: 10.1007/BF00914697.

34. Marangoni C. Sull espansione delle goccie di un liquido galleggiante sulla superficie di altro liquid. - Pavia : Tipografia dei fratelli Fusi, 1865. 